\title{
Monodromy in the Quantum Spherical Pendulum
}

\author{
V. Guillemin ${ }^{1}$ and A. Uribe ${ }^{2}$ \\ 1 Mathematics Department, M.I.T., Cambridge, MA 02139, USA \\ 2 Mathematics Department, Princeton University, Princeton, NJ 08544, USA
}

\begin{abstract}
In this article we show that monodromy in the quantum spherical pendulum can be interpreted as a Maslov effect: i.e. as multi-valuedness of a certain generating function of the quantum energy levels.
\end{abstract}

1.

A classical dynamical system of $n$ degrees of freedom is completely integrable if it admits $n$ independent integrals of motion, $H_{1}, \ldots, H_{n}$, with the property $\left\{H_{i}, H_{j}\right\}=0$. The standard method for integrating such a system is by means of action-angle variables. The action variables, $I_{1}, \ldots, I_{n}$ can, in principle, be computed by simple quadratures and the angle variables, $\theta_{1}, \ldots, \theta_{n}$ determined by the Darboux relation $\Omega=\sum d I_{i} \wedge d \theta_{i}$; and, in terms of them, the system takes the transparently simple form:

$$
\dot{\theta}_{i}=\frac{\partial H}{\partial I_{i}}\left(I_{1}, \ldots, I_{n}\right), \quad \dot{I}_{i}=0 .
$$

In [D] Duistermaat raised the question of whether Eq. (1.1), appropriately interpreted, make sense globally on the entire phase space of the system in question. He showed this to be the case iff certain topological invariants are zero. The most important of these is an invariant, called monodromy, which we will describe in Sect. 3. One integrable system for which it is non-zero is the spherical pendulum.

Recently Cushman and Duistermaat [C.D] detected monodromy effects in the quasi-classical behavior of the spherical pendulum. For the spherical pendulum the quasi-classical values of the energy and axial angular momentum form a set in the plane which looks like a twisted lattice. They show, however, that because of monodromy, it is impossible to construct an injective map of this set into the standard lattice, $\hbar \mathbb{Z}^{2}$, which scales properly as $\hbar \rightarrow 0$.

The question we will investigate below is: Can monodromy be detected in the quantum behavior of the spherical pendulum? More explicitly, consider the Schrödinger operator on $S^{2}$

$$
-\hbar^{2} \Delta+V
$$


$\Delta$ being the Laplace operator, $V$ the potential

$$
V(x, y, z)=z+C,
$$

and $C$ a constant which is sufficiently large to insure that $V$ is positive on $S^{2}$ (and hence that the spectrum of (1.2) lies entirely on the positive real axis). Since (1.2) is invariant with respect to rotations about the $z$-axis, it commutes with the operator

$$
-\sqrt{-1}\left(x \frac{\partial}{\partial y}-y \frac{\partial}{\partial x}\right) .
$$

The pair of operators, (1.2) and (1.4), define what we will from now on call the quantum spherical pendulum, and by its spectrum we will mean the set of all triples

$$
\left(\lambda_{1}, \lambda_{2}, \hbar\right)
$$

with the property that there exists a function on $S^{2}$ which is simultaneously an eigenfunction of (1.2) with eigenvalue $\lambda_{1}$ and an eigenfunction of (1.4) with eigenvalue $\lambda_{2}$. Given the data (1.5), one would like to know: Is it possible to "hear" the monodromy of the spherical pendulum in the sense that, for a region in the plane, one can "hear" its area and perimeter? Related to this is the question: Does monodromy have simple, detectible effects on the asymptotic behavior of the spectrum as $\hbar \rightarrow 0$ ? The first question turns out to be very easy to answer (in the affirmative, see Sect. 3). As for the second question, we have not been able to obtain the quantum analogue of the result in [C.D]; but we will show in Sect. 5 that certain generating functions of the spectral data (1.5) can be expressed in terms of action coordinates and hence are multivalued when the action coordinates are multi-valued.

One unpleasant feature of the system (1.2) and (1.4) is the dependence on $\hbar$. Therefore, rather than dealing directly with (1.2) and (1.4) we will replace them by the system of first-order self-adjoint operators on $S^{2} \times S^{1}$ :

$$
\begin{aligned}
& Q_{1}=\left(-\Delta-V \frac{\partial^{2}}{\partial \theta^{2}}\right)^{1 / 2} \\
& Q_{2}=-\sqrt{-1}\left(x \frac{\partial}{\partial y}-y \frac{\partial}{\partial x}\right), \\
& Q_{3}=-\sqrt{-1} \frac{\partial}{\partial \theta}
\end{aligned}
$$

$\theta$ being the angle variable on $S^{1}$. It is clear the spectrum of this system is essentially identical with the subset of $(1.5)$ consisting of $\left(\lambda_{1}, \lambda_{2}, \hbar\right)$ with $\hbar=1,1 / 2,1 / 3, \ldots$. The classical system associated with (1.6) is also essentially the classical spherical pendulum, though it appears at first glance to have three degrees of freedom rather than two. One of these is spurious, however, since the system does not depend directly on the "phoney variable" $\theta$. If one eliminates this variable and sets the momentum variable which is dual to it equal to $c$, one obtains a system of two degrees of freedom which is, up to rescaling by $c$, the standard classical spherical 
pendulum. For further details concerning this "homogeneity trick," see Sects. 6 and 7 of [G.U].

2.

We will begin with a few general observations about the functorial properties of symplectic manifolds. Given symplectic manifolds, $X$ and $Y$, a morphism

$$
X \stackrel{\Gamma}{\rightarrow} Y
$$

will, by definition, be a Lagrangian submanifold, $\Gamma$, of $X^{-} \times Y$ equipped with a half-density, $\sigma_{\Gamma}$. If $\Gamma: X \rightarrow Y$ and $\Theta: Y \rightarrow Z$ are morphisms, the composite morphism $\Theta \circ \Gamma: X \rightarrow Z$ is well-defined (see [D.G]) providing $\Gamma$ and $\Theta$ satisfy appropriate clean intersection hypotheses. Thus symplectic manifolds are "objects" in what one can, without too much poetic license, call the "symplectic category" (see [W.2]). Consider now the Hamiltonian action

$$
\tau: G \times X \rightarrow X
$$

of a Lie Group $G$ on a symplectic manifold, $X$, with moment mapping

$$
\Phi: X \rightarrow \mathrm{g}^{*} \text {. }
$$

The categorical way of describing (2.1) and (2.2) is by means of a morphism

$$
X \times X^{-} \stackrel{\Gamma_{\tau}}{\longrightarrow} T^{*} G
$$

This morphism will be called the moment morphism and is defined as follows: Imbed $X \times G$ in $X^{-} \times X \times G \times \mathfrak{g}^{*}$ by the mapping which sends $(x, g)$ onto $\left(x, \tau_{g} x, g, \Phi(x)\right)$. If one makes the identification of $G \times g^{*}$ with $T^{*} G$, this can be thought of as an embedding

$$
\gamma_{\tau}: X \times G \rightarrow X^{-} \times X \times T^{*} G
$$

Proposition 2.1 (Weinstein [W.1]). The image of $\gamma_{\tau}$ is a Lagrangian submanifold of the product symplectic manifold, $X^{-} \times X \times T^{*} G$.

If we equip $X$ with its intrinsic symplectic half-density and $G$ with (any) left-invariant half-density, then $X \times G$ acquires a product half-density which, via $\gamma_{\tau}$, gets identified with a half-density on the image of $\gamma_{\tau}$. This makes the image into a morphism in the sense above, and this is, by definition, the morphism (2.3). It clearly has built into it the data (2.1) and (2.2).

On the other hand there is a diagonal morphism

$$
\Delta:\{p t .\} \rightarrow X \times X^{-}
$$

consisting of $X$ (imbedded in $X \times X^{-}$via the diagonal imbedding) equipped with its intrinsic symplectic half-density. Under appropriate clean intersection hypotheses (see, for instance, Sect. 3), (2.4) and (2.3) can be composed, and one gets a morphism

$$
\Gamma_{\tau} \circ \Delta:\{p t .\} \rightarrow T^{*} G
$$


which consists of a Lagrangian submanifold, $\Lambda_{\tau}$, of $T^{*} G$ and a half-density, $\sigma_{\tau} . \Lambda_{\tau}$ is called the character Lagrangian of the action (2.1) and is the set

$$
\Lambda_{\tau}=\left\{(g, \xi) \in T^{*} G ; \exists x \in X, \tau_{g} x=x \text { and } \xi=\Phi(x)\right\} .
$$

The half-density $\sigma_{\tau}$ is a little more complicated to describe; however, we will compute it for a special class of $G$-actions in Sect. 4.

Now let $M$ be a compact manifold, and let $X$ be the cotangent bundle of $M$ with its zero section removed. Let $\tau$ be a homogeneous (or "canonical") action of $G$ on $X$; i.e. one which preserves the canonical one-form $\sum p_{i} d q_{i}$. We will say that $\tau$ is elliptic if the moment mapping, $\Phi: X \rightarrow \mathfrak{g}^{*}$, is proper; and we will define a quantization of (2.1) to be a unitary representation

$$
g \mapsto U_{g}
$$

of $G$ on $L^{2}(M)$ such that for each $g \in G, U_{g}$ is a unitary Fourier integral operator and $\tau_{g}$ its underlying canonical transformation. (Throughout this paper, by the way, $L^{2}$ will mean the space of $L^{2}$ half-densities rather than $L^{2}$ functions.) Assuming $\tau$ to be elliptic, it is easy to show that the expression

$$
\chi(g)=\operatorname{trace} U_{g}
$$

makes sense as a distributional function on $G$.

Proposition 2.2 (See [G.S.2]). The character, (2.8), of the representation (2.7) is a Fourier integral distribution with wave front set, $\Lambda_{\tau}$, and with symbol $\tau_{\tau}$.

One trivial but important consequence of this proposition is that $\Lambda_{\tau}$ and $\sigma_{\tau}$ are spectral invariants of the unitary representation (2.7), and, hence, so are any geometric objects associated with them (for instance, the action variables and monodromy invariants which we are going to describe below).

\section{3.}

Suppose now that $G$ is isomorphic to $\mathbb{R}^{n}$, where $n$ is the dimension of $M$. Suppose also that the action (2.1) is locally free except perhaps on a codimension-one subset of $X$. These assumptions imply that (2.1) is a completely integrable classical dynamical system, and so with some justification we can speak of the representation of $G$ on $L^{2}(M)$ as being a completely integrable quantum mechanical system. The goal of the next two sections will be to describe its character Lagrangian, $\Lambda_{\tau}$, and, at generic points of $\Lambda_{\tau}$, the half-density $\sigma_{\tau}$. This will require a review of some basic facts about the global structure of completely integrable systems. A good reference for this material is the first three sections of Duistermaat [D].

For the moment we will drop the assumption that $X$ is the cotangent bundle of $M$ and $\tau$ a "canonical" action. However, the other assumptions we made in the paragraph above will continue to be in force: namely we will assume $G \cong \mathbb{R}^{n}$, $2 n=\operatorname{dim} X, \tau$ locally free at generic points of $X$ and $\Phi: X \rightarrow \mathfrak{g}^{*}$ proper. Let $\mathfrak{g}^{0}$ be the set of regular values of $\Phi$. Since $\Phi$ is proper, $g^{0}$ is an open subset of $\mathrm{g}^{*}$ and its pre-image is a "large" $G$-invariant subset of $X: X-\Phi^{-1}\left(\mathfrak{g}^{0}\right)$ is closed and of codimension one. A theorem of Arnol'd says that over $\mathrm{g}^{0}$ 


$$
\Phi: X \rightarrow \mathfrak{g}^{0}
$$

is a fiber mapping, and each fiber is a finite union of Lagrangian tori. To simplify matters further, we will assume that the fibers of $\Phi$ are connected, in which case Arnold's theorem says that each fiber is a single Lagrangian torus on which $G$ acts transitively. Thus if $p \in \mathrm{g}^{0}$ and $q_{1}$ and $q_{2}$ are in $\Phi^{-1}(p)$, the stabilizer of $q_{1}$ in $G$ is the same as the stabilizer of $q_{2}$. We will denote this stabilizer group by $\Lambda_{p}$. Since $p \in \mathfrak{g}^{0}$, it is a discrete, co-compact subgroup of $G$; and, therefore, since $G \cong \mathbb{R}^{n}$, it is a lattice. Via the identifications

$$
T_{p}^{*} \cong \mathfrak{g} \stackrel{\exp }{\longrightarrow} G
$$

we can think of $\Lambda_{p}$ as a lattice sitting inside $T_{p}^{*}$; hence, as $p$ varies, we get a lattice bundle

$$
\Lambda \stackrel{P}{\rightarrow} \mathfrak{g}^{0}
$$

sitting inside $T^{*} \mathrm{~g}^{0}$. One of the main results of $[\mathrm{D}]$ is the assertion

Theorem 3.1. $\Lambda$ is a Lagrangian submanifold of $T^{*} g^{0}$.

Proof. Identifying $g$ with $G$ via the exponential map we get identifications

$$
T^{*} \mathfrak{g}^{0} \cong \mathfrak{g} \times \mathfrak{g}^{0} \cong G \times \mathfrak{g}^{0} \subset G \times \mathfrak{g}^{*} \cong T^{*} G .
$$

We leave for the reader to convince himself that, modulo these identifications, $\Lambda$ is the character Lagrangian associated with the action (2.1), i.e.

$$
\Lambda=\Lambda_{\tau} \text {. }
$$

Given this result, Duistermaat proceeds to define the "action variables" of the action (2.1) in the following fashion. Let $V$ be a simply connected open subset of $\mathrm{g}^{0}$. Then over $V$ the fibration

$$
\Lambda \stackrel{P}{\rightarrow} V
$$

is a trivial fibration with fibers $\mathbb{Z}^{n}$; and to each $n$-tuple of integers $\kappa=\left(\kappa_{1}, \ldots, \kappa_{n}\right)$ corresponds a section

$$
s_{\kappa}: V \rightarrow \Lambda \subset T^{*} V
$$

By the theorem the image of $s_{\kappa}$ is a Lagrangian submanifold of $T^{*} V$. Hence there exists a smooth real-valued function, $I_{\kappa}$, on $V$ with the property

$$
s_{\kappa}^{*} \alpha=d I_{\kappa},
$$

$\alpha$ being the canonical cotangent bundle one-form on $T^{*} V . I_{\kappa}$ is only determined up to an additive constant; however if we let $I_{1}, \ldots, I_{n}$ be the $I$ 's corresponding to the standard basis vectors we can take for $I_{\kappa}$ the sum

$$
I_{\kappa}=\kappa_{1} I_{1}+\cdots+\kappa_{n} I_{n}
$$

The functions $I_{1}, \ldots, I_{n}$ are, by definition, the action variables of the system (2.1). 
If $\mathrm{g}^{0}$ is simply connected, we can let the $V$ in the discussion above be $\mathrm{g}^{0}$ itself, in which case the action variables are globally defined. However, if $\mathfrak{g}^{0}$ is not simply connected, there is an obstruction to defining the action variables on all of $\mathfrak{g}^{0}$. Namely, the fundamental group of $\mathfrak{g}^{0}$ acts as deck transformations on the covering space, $\Lambda \rightarrow \mathrm{g}^{0}$. For a fixed base point, $p_{0} \in V$, these deck transformations permute the fibers above $p_{0}$; and thus give rise to a group homomorphism

$$
\pi_{1}\left(\mathfrak{g}^{0}, p_{0}\right) \stackrel{\rho}{\longrightarrow} \operatorname{Aut}\left(\Lambda_{p_{0}}\right) \cong G L(n, \mathbb{Z}) .
$$

Suppose now that $\gamma$ is a closed curve in $\mathfrak{g}^{0}$ with its initial point at $p_{0}$. If one analytically continues the $I_{i}$ 's around $\gamma$ in such a way that at each point, $p$, of $\gamma$, $\left(p,\left(d I_{i}\right)_{p}\right)$ is in $\Lambda$, then after reentry into $V, I_{i}$ has been converted into an expression of the form

$$
I_{i}^{\gamma}=\sum_{j=1}^{n} a_{i j}(\gamma) I_{j}+c_{i}(\gamma)
$$

where $a_{i j}(\gamma)$ is the $i-j^{\text {th }}$ entry of $\rho(\gamma)$ and the vector $c(\gamma)=\left(c_{1}(\gamma), \ldots, c_{n}(\gamma)\right)$ satisfies the cocyle condition

$$
c\left(\gamma_{1} \gamma_{2}\right)=c\left(\gamma_{2}\right)+\rho\left(\gamma_{2}\right) c\left(\gamma_{1}\right)
$$

Moreover, if we change $I_{i}$ to $I_{i}+c_{i}, c(\gamma)$ gets changed by the co-boundary, $\rho(\gamma) c$. The data $\rho$ and $c$ are, by definition, the monodromy invariants of the system (2.1), and their vanishing is obviously a necessary and sufficient condition for the existence of global action coordinates. It is known that they are non-vanishing for some very simple systems (the spherical pendulum, the Lagrange top). See [C.D].

\section{4.}

We now turn to the computation of the half-density $\sigma_{\tau}$, at regular points of $\Lambda_{\tau}$ (i.e. points, $(g, \xi)$ at which $\left.\xi \in \mathfrak{g}^{0}\right)$. Let $V$ be a simply-connected open subset of $\mathfrak{g}^{0}$, and $I_{1}, \ldots, I_{n}$ action variables on $V$. We will show that

$$
\sigma_{\tau}=(2 \pi)^{n} \frac{d I_{1} \cdots d I_{n}}{\left(d \xi_{1} \cdots d \xi_{n}\right)^{1 / 2}}
$$

at all points $(g, \xi) \in \Lambda_{\tau}$ for which $\xi \in V$ (the " $d \xi_{1} \cdots d \xi_{n}$ " in (4.1) being the restriction to $V$ of Lebesgue measure on $\mathrm{g}^{*}$ ).

Remark. The proof of (4.1) which we will give below is by no means the shortest proof we could give; however, it is (for us at any rate) the most illuminating. The notion of the "trace" of a symplectic mapping, which plays an important role in this proof, will be the subject of a sequel to this paper.

We will begin by making an assumption about $\tau_{g}$ at $(g, \xi)$ which will be in force throughout this section (but which we will eventually be able to get rid of). Since $\xi \in \mathfrak{g}^{0}$, the set

$$
X_{\xi}=\{x \in X ; \Phi(x)=\xi\}
$$


is a single $G$-orbit. Let $X_{g}$ be the fixed point set of $\tau_{g}$ in $X$. Since $(g, \xi) \in \Lambda_{\tau}$, this fixed point set is non-empty and by (2.6) it contains $X_{\xi}$.

Definition 4.1. We will say that $\tau$ satisfies the Birkhoff twist condition at $(g, \xi)$ iff: (i) $X_{\xi}$ is a connected component of $X_{g}$, and (ii) at every point $p \in X_{\xi}$, the tangent space to $X_{\xi}$ at $p$ is the fixed point set of the linear mapping $\left(d \tau_{g}\right)_{p}: T_{p} X \rightarrow T_{p} X$.

Remark. A fixed point set which satisfies condition (ii) is said to be clean (see [D.G]).

We will now give several alternative formulations of this condition. The simplest and easiest to state is the following:

Proposition 4.2. $\tau$ satisfies the Birkhoff twist condition at $(g, \xi)$ if and only if the cotangent fibration, $\pi: T^{*} G \rightarrow G$, maps a neighborhood of $(g, \xi)$ in $\Lambda_{\tau}$ diffeomorphically onto a neighborhood of $g$ in $G$.

We will prove this by giving two other formulations of the twist condition, both involving action coordinates. Fix a basis $e_{1}, \ldots, e_{n}$ of $\mathfrak{g}$, and let $\xi_{1}, \ldots, \xi_{n}$ be the coordinate functions on $\mathfrak{g}^{*}$ associated with these basis vectors. Identifying $\mathfrak{g}$ with $G$, we can think of the coordinates $x_{1}, \ldots, x_{n}$, dual to $\xi_{1}, \ldots, \xi_{n}$ as being a global system of coordinates on $G$. Finally let $V$ be a connected and simply connected open subset of $\mathfrak{g}^{0}$ containing $\xi$ and let $I_{1}, \ldots, I_{n}$ be a system of action coordinates on $V$. In Sect. 3 we proved:

Proposition 4.3. There exist $n$ integers, $\kappa_{1}, \ldots, \kappa_{n}$, such that near $(g, \xi), \Lambda_{\tau}$ is defined by the equations

$$
x_{i}=\frac{\partial I}{\partial \xi_{i}}, \quad i=1, \ldots, n,
$$

where $I=\kappa_{1} I_{1}+\cdots+\kappa_{n} I_{n}$.

As a corollary of this proposition we obtain:

Proposition 4.4. The cotangent projection $\pi: T^{*} G \rightarrow G$ maps a neighborhood of $(g, \xi)$ in $\Lambda_{\tau}$ diffeomorphically onto a neighborhood of $g$ in $G$ if and only if the Hessian determinant

$$
\operatorname{det}\left(\frac{\partial^{2} I}{\partial \xi_{i} \partial \xi_{j}}\right) \neq 0 \text { at } \xi .
$$

To relate (4.3) to the twist condition, we will make use of the "action-angle" theorem for completely integrable systems in the version given by Duistermaat in [D]. Since the $I_{i}$ 's are a system of coordinates on $V$, we can express the linear coordinates $\xi_{1}, \ldots, \xi_{n}$ in terms of them:

$$
\xi_{i}=H_{i}\left(I_{1}, \ldots, I_{n}\right), \quad i=1, \ldots, n .
$$

Now let $\theta_{1}, \ldots, \theta_{n}$ be angle coordinates on the $n$-torus

$$
T^{n}=S^{1} \times \cdots \times S^{1}
$$

and let $Q_{V}$ be the $2 n$-dimensional manifold

$$
Q_{V}=T^{n} \times V
$$


equipped with the symplectic form $\sum d I_{i} \wedge d \theta_{i}$. Finally, let $\mathbb{R}^{n}$ act on $Q_{V}$ by the action

$$
\tau_{a}(I, \theta)=\left(I, \theta+a_{1} \frac{\partial H_{1}}{\partial I}+\cdots+a_{n} \frac{\partial H_{n}}{\partial I}\right) .
$$

Proposition 4.5. (See Duistermaat, [D]). Let $X_{V}$ be the preimage of $V$ in $X$ with respect to the moment mapping $\Phi: X \rightarrow \mathrm{g}^{*}$, and identify $G$ with $\mathbb{R}^{n}$ by means of the coordinate system $x_{1}, \ldots, x_{n}$. Then there is an isomorphism of Hamiltonian G-spaces, $\gamma$ :

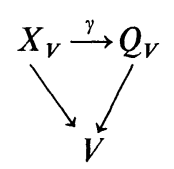

Corollary. If the coordinates of the point, $g$, in $G$ are $\left(a_{1}, \ldots, a_{n}\right)$ and of the point, $\xi$, in $V$ are $\left(I_{1}^{0}, \ldots, I_{n}^{0}\right)$, then $T^{*} G$ satisfies the Birkhoff twist condition if and only if

$$
\operatorname{det}\left(\frac{\partial^{2} H}{\partial I_{i} \partial I_{j}}\right) \neq 0 \text { at } I_{n}^{0}, \ldots, I_{n}^{0},
$$

where $H=a_{1} H_{1}+\cdots+a_{n} H_{n}$.

We will now show that (4.3) and (4.6) are the same condition. From the equation

we get

$$
x_{i}=\frac{\partial I}{\partial H_{i}}=\sum_{r=1}^{n} \kappa_{r} \frac{\partial I_{r}}{\partial H_{i}},
$$

$$
\kappa_{i}=\sum_{j=1}^{n} x_{j} \frac{\partial H_{j}}{\partial I_{i}}
$$

and hence (differentiating with respect to $I_{k}$ ):

$$
\sum_{j=1}^{n} \frac{\partial x_{j}}{\partial I_{k}} \frac{\partial H_{j}}{\partial I_{i}}+x_{j} \frac{\partial^{2} H_{j}}{\partial I_{k} \partial I_{i}}=0
$$

But

$$
\frac{\partial x_{j}}{\partial I_{k}}=\sum_{l=1}^{n} \frac{\partial x_{j}}{\partial H_{l}} \frac{\partial H_{l}}{\partial I_{k}}=\sum_{l=1}^{n} \frac{\partial^{2} I}{\partial H_{j} \partial H_{l}} \frac{\partial H_{l}}{\partial I_{k}}
$$

and the second term in (4.7), evaluated at $(g, \xi)$ is just the function

$$
\frac{\partial^{2} H}{\partial I_{k} \partial I_{i}}
$$

evaluated at $I_{1}^{0}, \ldots, I_{n}^{0}$. Hence Eq. (4.7), evaluated at $(g, \xi)$, just says that

$$
\sum_{j, l} \frac{\partial H_{j}}{\partial I_{i}} \frac{\partial^{2} I}{\partial H_{j} \partial H_{l}} \frac{\partial H_{l}}{\partial I_{k}}=-\frac{\partial^{2} H}{\partial I_{i} \partial I_{k}},
$$

proving the equivalence of (4.3) and (4.6) (and, in the process, proving Proposition 4.2).

Before turning to the proof of (4.1) we need to make a few comments about 
"traces" of symplectic mappings. To begin with, let $W$ be a symplectic vector space, and $S$ a linear symplectic mapping of $W$ onto itself. Then the fixed point set of $S$,

$$
\{w \in W ; S(w)=w\},
$$

possesses an intrinsically defined density (see [D.G], Lemma 5.1). We won't give the general recipe for this density here, but we will describe what this recipe yields in two special cases:

Example 1. Suppose (4.9) is a symplectic subspace, $U$, of $W$. Let $U^{\perp}$ be its symplectic orthocomplement, and let $A$ be the restriction of $S$ to $U^{\perp}$. Then the intrinsic density on $U$ given by the recipe above is:

$$
\frac{v_{U}}{|\operatorname{det}(I-A)|^{1 / 2}}
$$

where $v_{U}$ is the Liouville density associated with the symplectic form on $U$ (see the comments at the end of [D.G] Sect. 5).

Example 2. Suppose (4.9) is a Lagrangian subspace, $\Lambda$, of $W$. Then $I-S$ maps $\Lambda$ into zero and maps $W$ onto $\Lambda$; so it induces a bijective map

$$
A: W / \Lambda \rightarrow \Lambda \text {. }
$$

Let $\mu$ be a non-zero density on $\Lambda$ and let $v$ be the density on $W / V$ which is symplectically paired with it. Then $A^{*} \mu$ is a non-zero constant multiple of $v$. Let's call this multiple $\operatorname{det} A(\mu)$. Notice that if one replaces $\mu$ by $\lambda \mu$ (and hence $v$ by $(1 / \lambda) v)$ then

$$
A^{*}(\lambda \mu)=\lambda^{2}(\operatorname{det} A(\mu)) \frac{v}{\lambda},
$$

so $\operatorname{det} A(\lambda \mu)=\lambda^{2} \operatorname{det} A(\mu)$. Thus the product

$$
|\operatorname{det} A(\mu)|^{-1 / 2} \mu
$$

is independent of $\mu$. As might be expected this is the intrinsic density on $\Lambda$ yielded by the general recipe of [D.G], Lemma 5.1.

Now suppose that $X$ is a symplectic manifold and $f: X \rightarrow X$ a symplectic mapping with "clean" fixed points; i.e. suppose that the fixed point set, $X_{f}$, of $f$ is a submanifold of $X$ and that at every point, $p \in X_{f}$, the tangent space to $X_{f}$ is the fixed point set of the linear mapping

$$
d f_{p}: T_{p} X \rightarrow T_{p} X .
$$

Then by the result we have quoted ([D.G] Lemma 5.1), $T_{p} X_{f}$ has an intrinsically defined density which varies smoothly as one varies $p$ : in other words, $X_{f}$ itself is equipped with an intrinsically defined density, $\mu_{f}$. If $X_{f}$ is compact we define the integral

$$
S T(f)=\int_{X_{f}} \mu_{f}
$$

to be the symplectic trace of the mapping $f$ (or $S T(f)$ for short). 
We return now to the computation of $\sigma_{\tau}$. The definition of $\sigma_{\tau}$ which we gave in Sect. 2 involved the choice of an invariant half-density, $(d g)^{1 / 2}$, on $G$. In our case, $G$ being $\mathbb{R}^{n},(d g)^{1 / 2}$ is, up to a constant multiple, just the Lebesgue half-density on $G$.

Proposition 4.6. Let $\pi$ be the restriction to $\Lambda_{\tau}$ of the cotangent fibration, $T^{*} G \rightarrow G$. Then, at $(g, \xi)$,

$$
\sigma_{\tau}=S T\left(\tau_{g}\right)(d \pi)_{(g, \zeta)}^{*}(d g)^{1 / 2},
$$

providing $\tau$ satisfies the Birkhoff twist condition at $(g, \xi)$.

We won't give the proof of this result here, since (4.12) is a special case of a much more general result on symplectic traces which we will report on elsewhere. However, assuming (4.12), we will compute the right-hand side of (4.12) using Proposition 4.5. If $\left(a_{1}, \ldots, a_{n}\right)$ are the coordinates of $g$ in $G$, then by (4.5), $\tau$ is the mapping

$$
(I, \theta) \mapsto\left(I, \theta+\frac{\partial H}{\partial I}\right),
$$

where $H=a_{1} H_{1}+\cdots+a_{n} H_{n}$; and by (4.10)

$$
S T\left(\tau_{g}\right)=\int_{0}^{2 \pi} \cdots \int_{0}^{2 \pi}\left|\operatorname{det}\left(\frac{\partial^{2} H}{\partial I_{i} \partial I_{j}}\right)\right|^{-1 / 2} d \theta_{1} \cdots d \theta_{n} .
$$

Since the integrand does not depend on $\theta$, we end up with

$$
S T\left(\tau_{g}\right)=(2 \pi)^{n}\left|\operatorname{det}\left(\frac{\partial^{2} H}{\partial I_{i} \partial I_{j}}\right)\right|^{-1 / 2} .
$$

Using (4.8) the expression on the right can also be written as

$$
(2 \pi)^{n}\left|\operatorname{det}\left(\frac{\partial I_{i}}{\partial \xi_{j}}\right)\right|\left|\left(\frac{\partial^{2} I}{\partial \xi_{i} \partial \xi_{j}}\right)\right|^{-1 / 2},
$$

where $I=\kappa_{1} I_{1}+\cdots+\kappa_{n} I_{n}$. Since $(d g)^{1 / 2}=\left(d x_{1} \cdots d x_{n}\right)^{1 / 2}$ and $x_{i}=\partial I / \partial \xi_{i}$, we get the formula for $\sigma_{\tau}$ :

$$
\sigma_{\tau}=(2 \pi)^{n}\left|\operatorname{det}\left(\frac{\partial I_{i}}{\partial \xi_{i}}\right)\right|\left(d \xi_{1} \cdots d \xi_{n}\right)^{1 / 2},
$$

which can also be written more succinctly in the form (4.1). This concludes the proof of (4.1) if $\tau$ satisfies the twist condition at $(g, \xi)$. We will now show that this condition can be gotten rid of: Let $H_{i, r} i=1, \ldots, n$ be smooth functions on $V$. Using these we can define a Hamiltonian action of $\mathbb{R}^{n}$ on $Q_{V}$ by the formula

$$
\left(\tau_{r}\right)_{a}(I, \theta)=\left(I, \theta+a_{1} \frac{\partial H_{1, r}}{\partial I}+\cdots+a_{n} \frac{\partial H_{n, r}}{\partial I}\right)
$$

(compare with 4.5). Now choose the $H_{i, r}$ 's so that

(i) $H_{i, r} \rightarrow H_{i}$ as $r \rightarrow \infty$, and

$$
\operatorname{det}\left(\frac{\partial^{2}}{\partial I_{i} \partial I_{j}}\left(\sum_{k} a_{k} H_{k, r}\right)\right) \neq 0
$$


for all $r$, at the point $\left(a_{1}, \ldots, a_{n}, I_{1}, \ldots, I_{n}\right)$ corresponding to $(g, \xi)$. By what we have just proved, the formula (4.1) is true for $\tau_{r}$ at $(g, \xi)$; therefore, letting $r \rightarrow \infty$, it is true for $\tau$ as well.

\section{5.}

Now suppose that $M$ is a compact manifold, and (2.1) a homogeneous action of $G$ on $T^{*} M-\{0\}$. Let

$$
g \mapsto U_{g}
$$

be a unitary representation of $G$ on $L^{2}(M)$ by Fourier integral operators which is compatible with (2.1), and let

$$
\chi(g)=\operatorname{trace} U_{g}
$$

be its distributional character. By Proposition 2.2, $\chi$ is a Fourier integral distribution of order $n / 2$ with wave front set $\Lambda_{\tau}$ and symbol $\sigma_{\tau}$. Let $V$ be a simply connected open conic subset of $\mathfrak{g}^{0}$, and let $I_{1}, \ldots, I_{n}$ be action coordinates on $V$. In general, the $I_{i}$ 's are only determined up to additive constants; however, since $\tau$, is homogeneous, we can fix these additive constants by requiring that the I's themselves be homogeneous of degree one.

Let $\left(g_{0}, \xi_{0}\right) \in \Lambda_{\tau}$ with $\xi_{0} \in V$. Then by Proposition 4.3 there exists a linear combination, $I=\kappa_{1} I_{1}+\cdots+\kappa_{n} I_{n}$ of the $I$ 's with integer coefficients such that the equations

$$
x_{i}=\frac{\partial I}{\partial \xi_{i}}, \quad i=1, \ldots, n,
$$

$\xi \in V$ define an open conic subset of $\left(g_{0}, \xi_{0}\right)$ in $\Lambda_{\tau}$. By Hörmander's recipe for associating distributions with symbols (see $[\mathrm{H}]$ ), the character $\chi$ is equal on this conic set to the oscillatory integral

$$
(2 \pi)^{n} \int e^{\sqrt{-1}(x \cdot \xi-I(\xi))} d I_{1} \cdots d I_{n}+\cdots,
$$

the dots indicating terms of order less than the leading term (i.e. less than $n / 2$ ).

Notice that the measure $d I_{1} \cdots d I_{n}$ makes sense on all of $g^{0}$ whether monodromy exists or not, since the monodromy map (3.4) takes its values in the space of matrices of determinant \pm 1 . On the other hand, it is clear that the phase function in (5.4) is not globally defined on all of $\mathfrak{g}^{0}$ if there is monodromy; in other words:

Theorem 5.1. If monodromy is present it is impossible to represent the character of the representation (5.1) as a sum of simple oscillatory integrals of the form (5.4) globally on all $\mathfrak{g}^{0}$.

\section{References}

[C.D] Cushman, R., Duistermaat, J. J.: preprint (1988)

[D] Duistermaat, J. J.: On the existence of global action-angle variables. Commun. Pure Appl. Math. 33, 687-706 (1980)

[D.G] Duistermaat, J. J., Guillemin, V.: The spectrum of positive elliptic operators and periodic bicharacteristics. Invent. Math. 29, 184-269 (1975) 
[G.S.1] Guillemin, V., Sternberg, S.: Symplectic Techniques in Physics. New York: Cambridge University Press 1985

[G.S.2] Guillemin, V., Sternberg, S.: Homogeneous quantization and multiplicities of group representations. J. Funct. Anal. 47, 344-380 (1982)

[G.U] Guillemin, V., Uribe, A.: Circular symmetry and the trace formula, preprint (1988)

[H] Hörmander, L.: Fourier integral operators I. Acta Math. 129, 79-183 (1971)

[W.1] Weinstein, A.: Lectures on Symplectic Manifolds, CBMS Reg. Conf. Ser. Math., vol 29, Providence, R.I.: A.M.S. 1977

[W.2] Weinstein, A. Symplectic Geometry. In: Symposium on the Mathematical Heritage of Poincaré. Providence, R. I.: A.M.S. 1983

[W.2] Weinstein, A.: Symplectic Geometry. Bull. Am. Math. Soc. 5, 1-13 (1981)

Communicated by A. Jaffe

Received July 21, 1988 